\title{
Correction to: New Particle Formation and Growth Mechanisms in Highly Polluted Environments
}

\author{
Huan $\mathrm{Yu}^{1}$ (D) Lili Ren ${ }^{1} \cdot$ Vijay P. Kanawade ${ }^{2}$
}

(C) Springer Nature Switzerland AG 2019

\section{Correction to: Current Pollution Reports https://doi.org/10.1007/s40726-017-0067-3}

The original version of this article, unfortunately, contained an error. In the 2017 paper, "New Particle Formation and Growth Mechanisms in Highly Polluted Environments", there was an error in order of funders listed in the Acknowledgements. It should read:

Acknowledgements HY would like to thank the National Key Research and Development Program of China (2016YFC0203100), the National Science Foundation of China (grant nos. 41405116 and 41675124), the Natural Science Foundation of Jiangsu Province (grant no. BK20140989), and Jiangsu Specially Appointed Professor Grant. VPK would like to thank University Grants Commission, Government of India for Faculty Recharge Program Award (Ref. No. F.4-5(230-FRP/2015/BSR)).

The authors apologize for this error.

Publisher's Note Springer Nature remains neutral with regard to jurisdictional claims in published maps and institutional affiliations.

The online version of the original article can be found at https://doi.org/ $10.1007 / \mathrm{s} 40726-017-0067-3$

Huan Yu

hyu@nuist.edu.cn

$\triangle$ Vijay P. Kanawade

vijaykanawade03@yahoo.co.in

1 School of Environmental Science and Engineering, Nanjing University of Information Science and Technology, Nanjing, China

2 University Centre for Earth and Space Sciences, University of Hyderabad, Hyderabad, Telangana, India 